\title{
Editorial
}

\section{Acta Alternativa?}

In this issue of Acta Neuropsychiatrica we examine the 'alternative route' and consider the usefulness of some alternative and complementary therapies. The term complementary and alternative medicines (CAM) encompasses a whole array of treatments and interventions ranging from the widely recognized, such as acupuncture and St John's Wort, to the relatively obscure, eg polarity therapy. The term 'alternative' is broadly used to describe any form of treatment that substitutes for mainstream (usually Western) medicines, whereas the term 'complementary' is typically applied to treatments that are 'added on'. Patients with neuropsychiatric illnesses, such as depression and anxiety disorders, routinely trial CAM as evidenced by a recent study (1). Indeed, in the latest review (2) depression was noted as one of the most frequent indicators for using CAM, and this same article lists more than 50 complementary and alternative therapies. It is clear, that this is a field of medicine that cannot be ignored, if only for the simple reason that patients commonly use CAM and these have the potential to interact with conventional medicines. Perhaps the time has come for CAM to be viewed no longer as 'alternatives' but instead as mainstream, additional to standard therapy. However, this necessitates rigorous evaluation.

The efficacy and effectiveness of CAM is perhaps best assessed by considering the evidence base for each treatment or approach. However, evidence alone is unlikely to provide a complete answer as to why some clinicians prescribe CAM and why they have widespread uptake by patients. Clearly, the behavior of providers and consumers also needs to be examined so as to fully appreciate the context within which CAM are used.

In recent years there has been a notable resurgence of interest in CAM with significant uptake worldwide. One area of interest is meditation in its many forms and its effect on well-being. In this issue of Acta Neuropsychiatrica Ivanovski and Malhi (3) provide an in-depth critique of mindful- ness meditation in the treatment of affective, anxiety and personality disorders, examining in particular its psychology and neurophysiology. Clayton et al. (4) then review the literature pertaining to the neuropsychiatric use of omega-3 polyunsaturated fatty acids - especially in children and adolescents.

The evaluation of CAM using randomized controlled trial methodology is problematic. However, unless CAM can demonstrate significant effects in large trials with robust statistical designs, their professional adoption will be limited and largely reliant on 'belief in efficacy' (5).

Given that patients are constantly seeking alternatives to conventional medicine, perhaps we need to also consider alternatives to evaluating therapeutic efficacy.

Professor Gin S. Malhi
Department of Psychological Medicine
Northern Clinical School
University of Sydney
Sydney
Australia

\section{References}

1. Wilhelm K, Niven H, Mitchell P et al. Actions taken to cope with depression in patients seeking specialist care. Aust N Z J Psychiatry 2006;40:239-244.

2. Thachil AF, Mohan R, Bhurgra D. The evidence base of complementary and alternative therapies in depression. $\mathrm{J}$ Affect Disord 2007;97:23-35.

3. Ivanovski B, MaLhi GS. The psychological and neurophysiological concomitants of mindfulness forms of meditation. Acta Neuropsychiatr 2007;19:76-91.

4. Clayton E, Hanstock T, Garg $M$ et al. Long-chain omega-3 polyunsaturated fatty acids in the treatment of psychiatric illnesses in children and adolescents. Acta Neuropsychiatr 2007;19:92-103.

5. Hirschkorn KA, Bourgeault IL. Actions speak louder than words: mainstream health providers' definitions and behaviour regarding complementary and alternative medicine. Complement Ther Clin Pract 2007;13:29-37. 